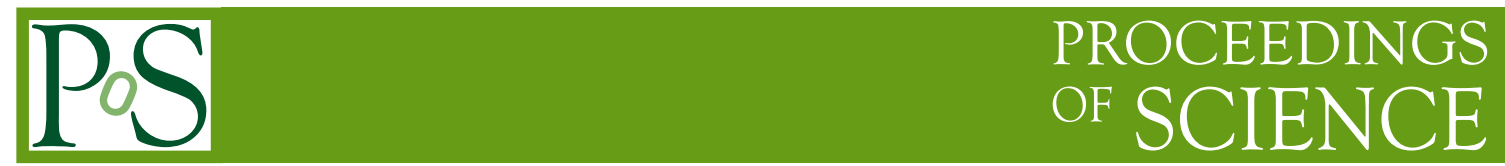

\title{
Challenges and progress on the SB horn design
}

\author{
MARCOS DRACOS* \\ IPHC/IN2P3 Strasbourg \\ E-mail: marcos.dracoseires.in2p3.fr
}

The hadron collection system is a key element in the neutrino beam production in the case of conventional or Super-Beam cases. For the CERN SPL to Fréjus 4 MW/50 Hz Super-Beam, the collection system, a magnetic horn, will be in very sever conditions never experienced in the past. A careful study of all elements of this system must be done taking into account that the target will be located inside the horn. These studies must integrate the characteristics of the corresponding proton driver and the new physics results. The whole system will be studied in the framework of the European project EUROv.

10th International Workshop on Neutrino Factories, Super beams and Beta beams June 30 - July 52008

Valencia, Spain

\footnotetext{
* Speaker.
} 


\section{Introduction}

Conventional muon neutrino beams are produced by the decay of mesons (pions and kaons). These mesons are produced by colliding a proton beam with a target. To send the neutrinos in the right direction, the only available possibility is to act on the direction of the charged mother particles. After the proton collision with the target, the emerging mesons are collected and focused towards the neutrino detector using a toroidal magnetic field. The hadron collector used very often in this application is a magnetic horn pulsed with a very high electrical current. In the case of the CERN SPL Super-Beam (SB) the operation conditions will be much more severe than in previous applications. Table 1 shows a comparison of some horns already used by past or ongoing projects. In this table one can see that the under investigation horn has a small length which could be an advantage during the fabrication and operation, but, on the other side, the proton driver power $(4 \mathrm{MW})$ and repetition rate $(50 \mathrm{~Hz})$ are considerably higher than other applications, a real challenge!

Table 1: Comparison of horns already used or under utilisation.

\begin{tabular}{|c|c|c|c|c|c|c|}
\hline Project & $\begin{array}{c}\text { Proton Energy } \\
(\mathrm{GeV})\end{array}$ & $\begin{array}{c}\text { Power } \\
(\mathrm{MW})\end{array}$ & $\begin{array}{c}\text { Rep. Rate } \\
(\mathrm{Hz})\end{array}$ & $\begin{array}{c}\text { Current } \\
(\mathrm{kA})\end{array}$ & $\begin{array}{c}\text { Number of } \\
\text { horns }\end{array}$ & $\begin{array}{c}\text { Length } \\
(\mathrm{m})\end{array}$ \\
\hline \hline CNGS & 400 & 0.2 & 2 pulses/6 sec & 150 & 2 & 6.5 \\
K2K & 12 & 0.0052 & 0.5 & 250 & 2 & $2.4-2.7$ \\
NUMI & 120 & 0.4 & 0.5 & 200 & 2 & 3 \\
MiniBoone & 8 & 0.04 & 5 & 170 & 1 & 1.7 \\
T2K & 50 & 0.75 & 0.3 & 320 & 3 & $1.4-2.5$ \\
SPL-SB & $3.5-5$ & 4 & 50 & $300-600$ & $1-2$ & 1.3 \\
\hline
\end{tabular}

An initial design of a horn prototype system (horn+reflector) [1, 2] foreseen for a neutrino factory (NF) has been made at CERN for a $2.2 \mathrm{GeV}$ proton beam. An optimisation and a redesign has been made in a SB context [3, 4], driven by the physics case of a long baseline experiment $(130 \mathrm{~km})$ between CERN and Fréjus (MEMPHYS detector location). From these studies, it came out that the optimal proton energy is between 3.5 and $4.5 \mathrm{GeV}$. The circulating electrical current is then required to be $300 \mathrm{kA}$ in the horn and $600 \mathrm{kA}$ in the reflector enveloping the horn (Fig. 1). Above these energies, the muon neutrino beam starts being contaminated by electron neutrinos mainly coming from kaon decays. Both studies concluded that the proton target has to be installed inside the horn to maximise the hadron collection. For the power dissipation of the system, this condition (coming from the relatively low proton energy and the consequently low forward hadron boost) imposes a very sever constraint. Mainly for this reason, the target/horn integration has to seriously be studied since the beginning. Although multi-physics simulation of the whole system could greatly help the conception of a reliable design, a dedicated $R \& D$ and testing with a target will be needed to validate these studies but also to face the various safety aspects (chemistry of heavy metals, high radiation levels, high voltage, high current...), which would also include the design of a complete remote handling installation for the horn and target maintenance and possible exchange.

The European FP7 Design Study EUROv will study all aspects of feasibility of the target, horn and integration of the two objects. 


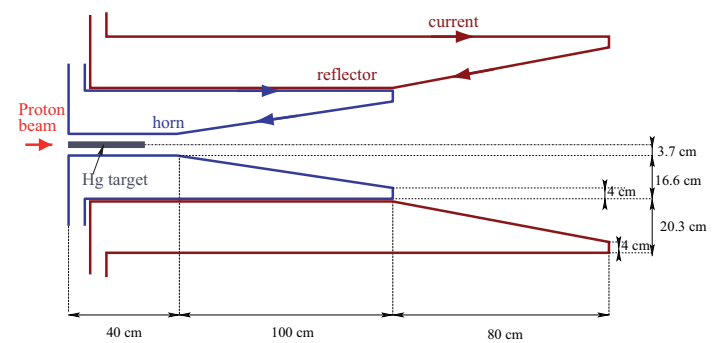

Figure 1: Schematic view of the horn and reflector optimised for a $3.5 \mathrm{GeV}$ proton beam.

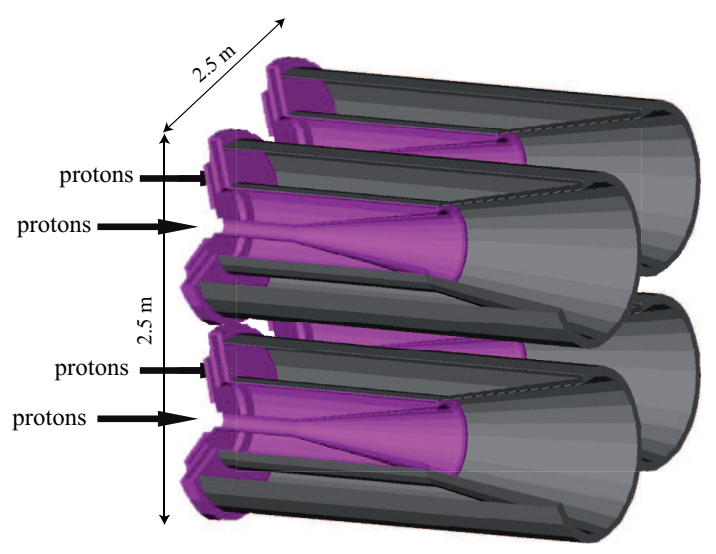

Figure 2: Schematic view of 4 target/horn systems sharing the proton beam power.

\section{Description}

The magnetic horn under study will have to focus hadrons (mainly pions) with a mean momentum of $600 \mathrm{MeV} / \mathrm{c}$ parallel to the beam axis and towards a distant detector. In the so called SB baseline of the project, the pions are produced by the impact of a primary $4 \mathrm{MW} / 3.5 \mathrm{GeV} / 2 \mathrm{~mA}$ pulsed proton beam delivered by a linac (SPL [5]) on a target located inside the horn (Fig. 1). The horn is composed by an aluminium internal and external as thin as possible skins $(<3 \mathrm{~mm})$ to minimise the energy deposition by the particles coming out of the target. To obtain the toroidal magnetic field needed for the hadron focusing, a high current circulates between the internal and external skins inducing inside the horn a magnetic field varying like $1 / r$ where $r$ is the distance from the horn axis. In this way, the magnetic field outside the horn completely vanishes in order the particles coming out to suitably stop spiring at the moment where their direction is parallel to the horn axis.

The electrical current needed to obtain this result is of the order of several hundreds of kA excluding the use of continues current sources because of the huge current consumption and power to be dissipated (otherwise the whole collection system has to be cryogenic with many other complications, see hereafter). Thus, the horn is submitted to a strong electromagnetic pulse producing thermo-mechanical stresses, vibrations, and fatigue. This project will benefit from the experience of CERN (prototype horn designed for $2.2 \mathrm{GeV}$ ), Miniboone, CNGS and T2K horns. The power supply design is an extrapolation of existing facilities and a first prototype version at only a fraction of the nominal current and repetition rate has been built and tested $(30 \mathrm{kA} / 1 \mathrm{~Hz})$ at CERN.

The horn shape strongly depends on the hadron energy and thus on the primary proton beam energy. Since the CERN design, the physics requirements have changed according to recent physics results and the actual required proton energy is of the order of $3.5 \mathrm{GeV}$ instead of $2.2 \mathrm{GeV}$. Mainly for this reason and to profit of new technological developments, a new horn design and prototype have to be done. An optimized horn design maximizing the neutrino beam intensity could help to reduce the challenging proton beam intensity or to reduce the experiments duration by few years.

A common proton driver solution with the same energy for SB and NF is under investigation. A $5 \mathrm{GeV}$ proton beam could be a good compromise for these two facilities (physics requires low 
energy protons for SB while going higher in energy would provide more pions for NF). But, the pion yield by 3-20 GeV protons is however poorly known. Geant 4 and MARS simulations give significantly different results (up to 50\%) in the best phase space regions both for super-beams and neutrino factories. HARP experiment has recently published results [6] showing that the pion yield is maximum for protons between 5 and $8 \mathrm{GeV}$ (for at least few different targets) pushing to conclude that a proton driver of $5 \mathrm{GeV}$ energy could be a good compromise between SB and NF.

In this case, the horn shape has to be re-optimised to take into account the new hadron transverse momentum and tune the neutrino energy distribution and intensity according to the desired physics performance of the facility.

\section{Horn Pulsing System}

A very important issue of the focusing system is the design of the horn pulser which also could limit the whole system's lifetime. This device is mainly composed of a capacitive energy bank able to deliver an energy of about $35 \mathrm{~kJ}$, a charger and a commutation and energy recuperation system. Indeed, thanks to the low power dissipation inside the horn due to its low resistance, theoretically up to $80 \%$ of the injected energy could be recuperated and reinjected to the system making substantial economies on the power budget. The study of this recuperation option will constitute a significant part of this project.

The capacitive energy bank consisting of several capacitors will store the energy received by the charger (positive polarity) and deliver it to the load when the appropriate switch is closed. Due to the oscillating circuit, a negative voltage will be applied to the capacitors after the pulse, providing thus some electrical energy which could be reused before next pulse but after voltage reversal. The pulsing circuit is the most challenging part of the pulsing system. It will produce the short pulses for the load and manage the negative voltage remaining after the pulses in order to reverse the polarity and recover some energy.

To study CP violation with the corresponding experiment, neutrinos and anti-neutrinos are needed. Some ideas have been expressed concerning the horn operation, where, instead of having dedicated periods to one neutrino kind, to mix both periods by sending in the horn alternatively a positive and negative pulses. In this way, after the pulse oscillating signal changes "naturally" polarity, instead of using switches to revert this polarity, the charger would add to the pulse the missing power (due to dissipation in the system) and send an opposite polarity pulse to the horn. This option, under investigation, needing less switches, will probably extent the lifetime of the system.

\section{Integration Issues}

Due to the very sever operation conditions, the whole system's integration including the target, the horn, the pulser and the cooling system, has to be carefully studied. Liquid targets as mercury studied for NF and presenting less power dissipation problems would add extra difficulties in this application mainly due to the small size of the internal part of the horn. Solid targets, used up to now are not able to cope with the $4 \mathrm{MW}$ beam power. Recent studies show that the maximum beam energy using solid targets could not exceed $2 \mathrm{MW}$. 
To be able to use solid targets, one could use 4 systems as depicted by Fig. 2. This takes advantage of the small horn size and from the reduced length of the hadron decay tunnel $(\sim 50 \mathrm{~m})$ just after the horn which diameter can be adjusted to satisfy the 4 horn system. In this case, the proton beam power for each target/horn system is reduced to $1 \mathrm{MW}$. This scheme presents other advantages as less exposure to radiation and easier power dissipation. The main disadvantage comes from the beam sharing. To send the proton beam in the 4 systems, 4 proton lines will be needed pulsed simultaneously (with $1 / 4$ th of the total proton power) or one after the other (with the totality of the proton power). These 4 beam lines will add a non negligible extra cost to the proton beam facility. To avoid this problem one could envisage a rotating 4 target/horn system as the one of Fig. 2 or a more linear translating system where the target/horn systems are on a straight line. In these last 2 cases, it is not any more needed to increase the diameter of the decay tunnel.

The 4 target/horn system doesn't reduce the operation load of the horn pulsing system which has always to be operated with the same high current and repetition rate. One possibility to reduce these problems too and probably all the target/horn cooling issues, is to use a cryogenic horn. Instead of using a metallic skin for the horn, the shape of the horn could be reproduced by a supraconducting $1 \mathrm{~mm}$ diameter wire (toroidal coil). The horn wire could be in a thin envelop containing superfluid $\mathrm{He}$, while outside, a gas He could be circulated to avoid quenching problems. If this very ambitious option becomes possible, no pulsed power supply will be needed for the horn but just DC current. To reduce the length of the proton pulse from $0.57 \mathrm{~ms}$ to few $\mu \mathrm{s}$ (affordable by the current pulse duration sent to the horn), after the proton driver, an accumulator and a compressor ring is normally foreseen [5]. Thanks to the DC current, this would not anymore be needed in the case of a cryogenic horn which constitutes a big advantage and would be a good reason to include this study in EUROv project.

Due to the absence of testing facilities, in this $R \& D$, the role of simulation is very important. It can enable the testing of different design concepts, reduce the experimental working load and provide a more cost-effective engineering of the system. Experiences from other academic and industrial fields such as RF cavity (modelling, design, tuning) and induction heating will be integrated in order to minimize the time to reach a viable solution. A tuning of these simulations (fatigue, deformations, modal analyses, transient thermo mechanical excitation of the structure, skin effect and Joule heating, power dissipation, heat exchange and cooling, radiation resistance, etc.) could be done using input provided by the previously described tests and facilities.

\section{References}

[1] A. Ball et al. CERN-NUFACT-Note-42.

[2] S. Gilardoni, PhD Thesis, Université de Genève, 2004 (http://doc.cern.ch/archive/electronic/cern/preprints/thesis/thesis-2004-046.pdf).

[3] J.E. Campagne CERN-NUFACT-Note-138.

[4] A. Cazes, PhD Thesis, Université Paris VI, 2004 (http://tel.ccsd.cnrs.fr/tel-00008775/en/).

[5] "Design of the SPL II", CERN-2006-006, 12 July 2006.

[6] M.G. Catanesi et al., Eur. Phys. J. C 51, 787-824 (2007). 